\title{
Special issue on argumentation in multi-agent systems
}

\author{
Katie Atkinson ${ }^{\mathrm{a}}$, Federico Cerutti ${ }^{\mathrm{b}, *}$, Peter McBurney $^{\mathrm{c}}$, Simon Parsons ${ }^{\mathrm{c}}$ and Iyad Rahwan ${ }^{\mathrm{d}}$ \\ ${ }^{a}$ University of Liverpool, $U K$ \\ ${ }^{\mathrm{b}}$ Cardiff University, $U K$ \\ ' King's College London, UK \\ ${ }^{\mathrm{d}}$ Massachusetts Institute of Technology, USA
}

\section{Introduction}

Different agents within a multiagent system (MAS) potentially have access to different information and different capabilities (including reasoning capabilities), different beliefs about the world, different preferences, constraints, and desires, and different goals. A key aspect of the scientific and engineering study of multiagent systems, therefore, has been the development of methods and procedures for identifying, assessing, reconciling, arbitrating between, managing, and mitigating such differences. Market mechanisms and voting procedures are two classes of methods for dealing with such differences. Argumentation is another, and it has long been recognised ${ }^{1}$ that argumentation can be helpful in this context. Argumentation can be understood as the formal interaction of different arguments for and against some conclusion, for example, a proposition, a preference, or an intention to underake some action. An agent may use argumentation techniques to perform individual reasoning itself, alone, in order to resolve conflicting evidence, or to decide between conflicting goals it may have. Two or more agents may also jointly use dialectical argumentation to identify, express, and reconcile differences between themselves, by means of interactions such as negotiation, persuasion, inquiry and joint deliberation.

By 2004, argumentation was well established as an important technology within the field of multiagent systems, particularly within the area of agent communication, and much work on dialectical forms of argumentation was published in this context. However, while papers on argumentation were well represented at more general conferences such as the International Conference on Autonomous Agents and Multiagent Systems (AAMAS) and AI \& Law, there was no specialist meeting on computational

\footnotetext{
${ }^{*}$ Corresponding author. E-mail: ceruttif@ cardiff.ac.uk.

${ }^{1}$ A quick search backs up the recollection that the earliest paper to discuss the use of argumentation in a multiagent system made up of interacting software entities is "Resolving goal conflicts via negotiation" by Katia P. Sycara, in: Proceedings of the 7th National Conference on Artificial Intelligence, pp. 245-250, St. Paul, MN, 1988.
} 
argumentation (and, for that matter, no specialist journal). In response this situation, Iyad Rahwan persuaded several of us to support the notion of a workshop, to be called Argumentation in MultiAgent Systems (ArgMAS) to be held in conjunction with AAMAS in New York City, USA in 2004. The first workshop was chaired by Iyad in conjunction with Pavlos Moraitis and Chris Reed, and the three of them formed the organising committee along with Antonis Kakas, Nicolas Maudet, Peter McBurney and Simon Parsons.

Subsequent workshops were held in conjunction with further editions of AAMAS, in Utrecht, The Netherlands (2005), Hakodate, Japan (2006), Honolulu, USA (2007), Estoril, Portugal (2008), Budapest, Hungary (2009), Toronto, Canada (2010), Taipei, Taiwan (2011), Valencia, Spain (2012), St. Paul, Minnesota, USA (2013), Paris, France (2014) and Istanbul, Turkey (2015). This global diversity was reflected in the presenters, invited speakers, and participants who similarly came from countries around the world. Through the 12 years that ArgMAS was running, the computational argumentation community grew steadily in strength, and now has its own dedicated conference, Conference on Computational Models of Argument (COMMA), and its own journal, Journal of Argument and Computation. This growth of argumentation community's events led inevitably to subsume the AAMAS workshop, and, reluctantly, the organising committee decided that the 2015 edition of ArgMAS would be the last.

The papers from ArgMAS 2004 until 2011 were published as edited volumes, and we decided to mark the end of the workshop series by publishing a collection of the best papers from the final years of the workshop. To that end, this special issue contains extended and carefully reviewed versions of contributions discussed in from the ArgMAS editions from 2012 to 2015 (inclusive). The papers passed a first peer-review process to be accepted into an ArgMAS workshop, and then another peer-review process to be included in this special issue. The papers in this volume are as follows:

In "A functional perspective on argumentation schemes," Adam Wyner presents a syntactic analysis that integrates argumentation schemes with abstract argumentation in order to derive a computational approach so that agents can use the components of a scheme to construct and present arguments and counterarguments. The result is a functional language for a computational analysis of argumentation schemes that is compatible with argumentation frameworks.

Katie Atkinson and Trevor Bench-Capon, in "States, goals and values: Revisiting practical reasoning," extend proposals concerning an argumentation scheme for practical reasoning grounded on action-based alternating transition systems augmented with values to enable the proper representation of, and ability to reason with, goals. This provides the explanation facilities associated with argumentation in general and value-based argumentation in particular, which are harder to obtain if a black box setup such as that in common utility-based approaches is used.

On a complementary topic, Douglas Walton, Alice Toniolo, and Timothy J. Norman, in "Towards a Richer model of deliberation dialogue: Closure problem and change of circumstances," explore the problem of whether models for agent deliberation capture the richness of human deliberative dialogue. The authors show that existing proposals represent good models of how deliberation proceeds in real settings, and they extend this work to consider changes in circumstances during the course of the dialogue, thus allowing the exchange of new knowledge.

Considering more general communication strategies, in "Assessing communication strategies in argumentation-based negotiation agents equipped with belief revision" Ana Casali, Pablo Pilotti, and Carlos Chesñevar consider the case of argumentation-based negotiation agents equipped with belief revision operations in the generation and interpretation of arguments. Because the belief revision process depends on the information the agents exchange in their utterances, the authors focus on different communication strategies the agents may implement and the impact these have in the negotiation process. 
From a more applied perspective, in "Firewall configuration: An application of multiagent metalevel argumentation" Andy Applebaum, Zimi Li, Karl Levitt, Simon Parsons, Jeff Rowe, and Elizabeth I. Sklar examine the use of a system of metalevel argumentation for firewall configuration. They show how their approach goes in the direction of an explainable artificial intelligence by making conflicts and their origins clear, and how different instantiations of a metalevel argumentation system provide alternative ways to resolve conflicts.

We thank the peer-reviewers who assessed the papers for this special issue and we thank the journal editors for their continued support. Considered together, these papers show the diversity of research and applications in the field of argumentation in multi-agent systems, and reveal the intellectual challenges inherent in this exciting domain. We commend the special issue to you.

Katie Atkinson, University of Liverpool, UK Federico Cerutti, Cardiff University, UK

Peter McBurney, King's College London, UK

Simon Parsons, King's College London, UK Iyad Rahwan, Massachusetts Institute of Technology, USA

\section{List of reviewers for the special issue}

From an editor's perspective, ${ }^{2}$ the unsung heroes of any endeavour in academic publishing are the reviewers. For once, here we get to celebrate those without whom this issue would not have been possible:

Ofer Arieli, The Academic College of Tel-Aviv, Israel

Pietro Baroni, Università degli Studi di Brescia, Italy

Floris Bex, Utrecht University, The Netherlands

Elise Bonzon, LIPADE, Université Paris Descartes, France

Richard Booth, Cardiff University, UK

Federico Cerutti, Cardiff University, UK

Carlos Chesñevar, Universitad Nacional del Sur, Argentina

Sylvie Coste-Marquis, CRIL, CNRS - Université d'Artois, France

Madalina Croitoru, University Montpellier II, France

Sofie De Clercq, Ghent University, Belgium

Wolfgang Faber, University of Huddersfield, UK

Dov Gabbay, King's College London, UK

Sebastian Gottifredi, Universitad Nacional del Sur, Argentina

Stella Heras, Universitat Politècnica de València, Spain

Magdalena Kacprzak, Białystok University of Technology, Poland

Gabriele Kern-Isberner, Technische Universität Dortmund, Germany

Beishui Liao, Zhejiang University, China

Jean-Guy Mailly, Technische Universität Wien, Austria

Nicolas Maudet, LIP6, Université Pierre et Marie Curie, France

Sanjay Modgil, King's College London, UK

Maxime Morge, Université de Lille, France

\footnotetext{
${ }^{2}$ Of course, from an author's perspective, reviewers often look rather different.
} 
Timothy Norman, University of Southampton, UK

Nir Oren, University of Aberdeen, UK

Jörg Pührer, Leipzig University, Germany

Simon Parsons, King's College London, UK

Ariel Rosenfield, Bar-Ilan University, Israel

Claudia Schulz, Imperial College London, UK

Mark Snaith, University of Dundee, UK

Hannes Strass, Leipzig University, Germany

George Theodorakopulos, Cardiff University, UK

Francesca Toni, Imperial College London, UK

Alice Toniolo, University of St. Andrews, UK

Paolo Torroni, Università di Bologna, Italy

Wamberto Vasconcelos, University of Aberdeen, UK

Bart Verheij, University of Groningen, The Netherlands

Johannes Wallner, University of Helsinki, Finland 